24. Schlegel: Aktinomykose. Ergebnis der allgemeinen Pathologie, Wiesbaden, 1900.

25. Hoche: Histogenèse du nodule actinomycotique. A. d. med. exp., 1899.

26. Wildermuth: Citiert bei Boström.

27. Pawlowsky et Maksutoff: Phagocytose dans l'actinomycose. Ann. de l'instit. Paris, 1893.

28. Ullmann: Beitrag zur Lebre von der Aktinomykose. Wiener med. Presse, 1888.

29. Rabe, Baug, Kitt: Citiert bei Schlegel.

30. Partsch: Die Aktinomykose des Menschen. Sammlung klin. Vorträge, No. 306, 307, 1888.

31. Korángi: Nothnagels specielle Pathologie und Therapie, Bd. 5, Th. 5. 32. Goldmann, Kanter: Citiert bei Ehrlich und Lazarus, Nothnagels spezielle Pathologie und Therapie, Bd. 8, Teil I.

\title{
XVI.
}

\section{Ein Fall von Aktinomykose der Lunge, der Leber ind des Herzens beim Menschen.}

\author{
Von \\ Dr. Gustav Fütterer in Chicago, Ill.
}

(Mit einer Textfigur.)

Die Gründe, welche mich veranlaßten, diesen Fall zu veröffentlichen, sind folgende:

1. Alle Fälle von Aktinomykose des Menschen sollten der Literatur hinzugefügt werden.

2. Die Diagnose wurde intra vitam gestellt.

3. Das Herz war sekundär beteiligt.

4. Die Beobachtung des aktinomykotischen Peribronchitis erlaubte einen nützlichen Vergleich mit tuberkulöser Peribronchitis.

Der Fall, welchen ich beschreibe, wurde vom Herrn Dr. Buchsin dem Herrn Dr. A. J. Ochsner überwiesen, welcher intra vitam die Diagnose auf Aktinomykose stellte. Die Krankengeschichte, für welche ich Herrn Dr. A. J. Ochsner zu Dank verpflichtet bin, lautet wie folgt: 
Aufnahme am 18. Dezember 1898. Entlassung am 19. Dezember 1898. Diagnose: Aktinomykose der Bauchdecken.

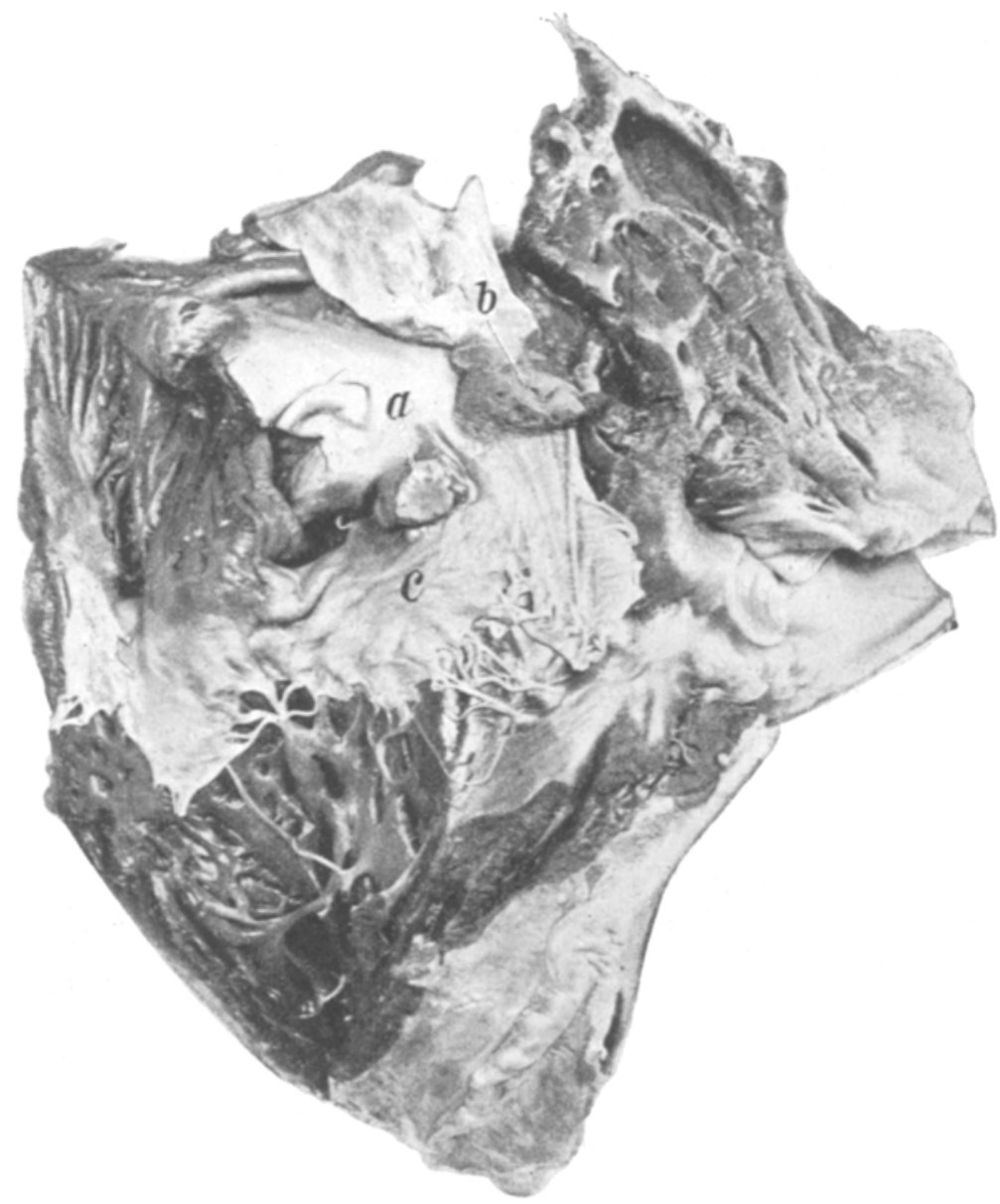

Aktinomykose des Herzens, das sich im Zustande der braunen Atrophie befindet. a und b zwei aktinomykotiscbe Knötchen, welche von schwefelgelber Farbe waren und die Strahlenpilze enthielten. c Tricuspidalklappe.

Krankengeschichte No. 5661. Resultat: Gebessert. Behandelnder Arzt: Dr. A. J. Ochsner. Name, B.; Alter 39 Jahre; Nationalität, Amerikaner; Beruf: Handlungsreisender. Familiengeschichte: Mutter 
starb an Krebs des Pankreas, 57 Jahre alt. Vater starb im Alter von 35 Jahren, Todesursacbe unbekannt. Der einzige Bruder lebt und ist gesund. Persönliche Geschichte: Patient hatte die gewöhnlichen Kinderkrankheiten, war aber sonst gesund; er verbeiratete sich im Alter von 24 Jahren und hat ein Kind, das 17 Jahre alt und gesund ist. Keine Syphilis.

Vor 7 Jahren stellten sich starke Schmerzanfälle im Epigastrium ein, die gewöhnlich von Brechen begleitet waren. Die Schmerzen danerten von $\frac{1}{2}$ bis 5 Stunden, worauf er von $\frac{1}{2}$ bis 15 Tage das Bett hüten mußte.

Im Laufe von 3 Jahren wiederbolten sich die Schmerzanfälle ungefäbr achtmal. Verschiedenemale wurde leichte Gelbsucht bemerkt und einmal war dieselbe sehr stark entwickelt. Vor 4 Jahren wurde eine Operation vorgenommen. Es wurde eine Inzision in der Regio hypochondriaca dextra gemacht, wobei eine käsige Masse gefunden und entfernt wurde. Heilung per primam. Einen Monat später wurde wieder eine Laparotomie vorgenommen, wobei peritonitische Adhäsionen und wieder eine käsige Masse gefunden wurden. Die Wunde heitte dieses Mal langsam und brach dann hin und wieder auf, etwas Eiter entleerend. Bis vor einem Jahre war das Abdomen nicht schmerzhaft, doch stellte sich dann ein Wundgefühl am oberen Ende der Narbe ein. Es entwickelte sich eine Schwellung, welche geöffnet wurde, worauf sich eine Fistel bildete, von welcher stetig Eiter abgeschieden wurde. Während des letzten Jahres waren abends Fiebertemperaturen vorhanden und während der letzten drei Monate sehr viel Husten und Expektoration.

Status praesens: Ziemlich gut genährt. Gesicht gerötet, Zunge belegt; Puls 92, regelmäßig, leicht unterdräckbar; Appetit schlecht, Stuhlverstopfung. Die Regio supraclavicularis beiderseits etwas eingezogen. Ausdehnung der linken Lunge gut, rechts gering. Relative Dämpfung über der rechten Spitze vorn und verschärftes vesikuläres Atmen an dieser Stelle. Verlängertes Expirium, keine Rasselgeräusche. Leichte Skoliose im dorsalen Teile, nach rechts. Über dem Abdomen eine groBe Anzahl pigmentierter Flecken und zwei große Narben, von denen die eine vom Knorpel der 11. Rippe rechts bis halbwegs zur Symphysis ossis pubis verläuft, während die andere vom Knorpel der 8. Rippe links ebenfalls halbwegs zur Symphyse hinabreicht. Am oberen Ende der zweiten Narbe, der Mittellinie zu, befinden sich zwei von Schorfen und Krusten geschlossene Fistelöffnungen. Im Epigastrium Empfindlichkeit, aber keine Dämpfung.

Behandlung: Die Fisteln wurden sondiert, und Gänge, welche in verschiedene Richtungen 'führten, wurden gefunden, geöffnet und ausgeschabt. Einer der Gänge verlief bis zum Peritonaeum. Das ausgeschabte Material enthielt kleine gelbeKörnchen, in denen die charakteristischen Strahlenpilze gefunden wurden. Das Sputum wurde wiederholt auf Tuberkelbazillen und Strahlenpilze untersucht, aber es wurden keine gefunden. 
Jodkaliumbehandlung brachte zeitweilige Besserung. Am 10. Oktober 1899 starb der Kranke.

Sektion am 11. Oktober 1899 (Fütterer).

Am Abdomen finden sich die oben beschriebenen Narben und nach der Eröffnung der Bauchböhle zeigen sich Adhäsionen zwischen Bauchwand und linken Leberlappen, aber bedeutend mehr über dem rechten Leberlappen, der sehr vergröBert ist. In der Bauchhöble eine Menge k]arer, grüner Flüssigkeit. Die Peric ardhöhle enthält wenig klare Flüssigkeit. Das Herz ist klein, die Gefäße an seiner Oberfläche zeigen gewundenen Verlauf, das viscerale Perikardium ist geschrumpft und statt des subepicardialen Fettes ist eine schwappende schleimige Masse vorhanden. Das Herzfleisch schimmert dunkelbraun durch das Perikardium, seine Konsistenz ist fest und auf dem Durchschnitte ist es trocken und starr und von bräunlicher Farbe. Auch das Endokardium ist gescbrumpft. Der Klappenapparat ist intakt, doch finden sich an der Basis der Trikuspidalklappe zwei gelbe Knötchen (Fig. 1, a u. b), welche der Koronararterie seitlich aufsitzen. Knötchen a ist $0,9 \mathrm{~cm} \mathrm{lang,} 0,4 \mathrm{~cm}$ breit und 0,4 $\mathrm{cm}$ hoch; Knötchen $b$ ist $1 \frac{1}{10} \mathrm{~cm}$ lang, $\frac{1}{2} \mathrm{~cm}$ breit und $0,5 \mathrm{~cm}$ hoch; die Knötchen sind scharf umschrieben und bilden blumenbeetartige Erhebungen mit abgerundeter, leicht unregelmäBiger Oberfläche. Die Farbe der Knötchen ist ein reines Schwefelgelb und ihre Konsistenz ist ziemlich fest. Bei Eröffnung der Brusthöhle kollabieren beide Lungen nur unvollständig. Kein abnormer Inhalt in der linken Pleurahöble, auch keine Verwachsungen, ausgenommen an der Basis. Im oberen Teile der rechten Pleurahöhle etwas klare, grüngelbe Flüssigkeit, unterer Teil der Pleurahöhle völig obliteriert, sodaß die rechte Lunge in Verbindung mit dem Zwerchfell und der Leber entfernt werden mußte.

Linke Lunge: Mäßiger Grad von Anthrakosis, untere zwei Drittteile atelektatisch und hier und da über den größten Teil der Lunge verbreitet runde Knötchen, welche durchschnittlich $\frac{1}{2} \mathrm{~cm}$ Durchmesser haben; dieselben sind von gelber Farbe und fester Konsistenz.

Rechte Lunge: An der Basis eine narbige Partie, deren größter Durchmesser etwa $3 \mathrm{~cm}$ beträgt, die annähernd Keilform hat, von grauer Farbe ist und sehr feste Konsistenz zeigt; hieran schließen sich graurötliche pneumonische Herde in den anderen beiden Lappen. Mit dem $\mathrm{Z}$ werchfell sind Lunge and Leber auf das innigste verwachsen und im Innern der Verwachsungen, wie auch in den Geweben des Zwerchfells finden sich gelbe Pünktchen oder solche mit einem rostfarbenen Anfluge, welche absteigend immer zahlreicher werden und zuletzt das Zwerchfell durchbrechend in den rechten Leberlappen eindringen und bier wiederum ausgedehnte Veränderungen erzeugen, die nur wenig Lebersubstanz übrig lassen. Die erkrankten Teile zeigen ein Netz von grauschwarzen Linien, dessen Maschenräume von halbflüssigen, gelben Massen ausgefüllt sind, die feine orange- 
farbene Körnchen enthalten. Außerdem siebt man auch den Durchbruch gelber Herde durch das $Z$ werchfell mit Veränderungen der Leber in absteigender Richtung. An einigen Stellen finden sich zwischen. Zwerchfell und rechtem Leberlappen größere Abscesse. Die Gallenblase ist erweitert, ihre Wand verdickt. Inhalt: Trübe Galle und vier kleine Steine. Die retroperitonaealen Lymphdrüsen und auch die nahe der Porta hepatis gelegenen sind etwas geschwollen. Die übrigen Organe in der Bauchböhle zeigen keine besonderen Veränderungen. Die Mesenterial$\mathrm{drü} \operatorname{sen}$ sind nicht geschwollen.

Kehren wir zur rechten Lunge zurück, so finden wir hier und da bis zur Spitze hinauf gelbe peribronchitische Herde und ebensolche runde Knötchen, wie sie in der linken Lunge beschrieben wurden.

Die Untersuchung des Gehirns wurde nicht erlaubt.

Diagnose: Actinomycosis cordis, Pulmonis utriusque, Dia. phragmatis et hepatis.

Mikroskopische Untersuchung. Von allen erkrankten Teilen wurden Schnitte untersucht und überall wurden die charakteristischen Strablenpilze gefunden. Im Gallenblaseninhalt in den geschwollenen Drüsen der Bauchhöhle und im Innern der Gallensteine konnten keine Strahlenpilze nachgewiesen werden.

Die Peribronchitis actinomycotica: Es gill im allgemeinen für unmöglich, bei einfach makroskopischer Betrachtung zwischen aktinomykotischer und tuberkulöser Peribronchitis zu entscheiden, doch muß ich sagen, daß dieses durchaus nicht schwierig ist, wenn man die Eigentümlichkeiten beider Zustände genau vergleicht.

Bei der Peribronchitis actinomycotica werden die Durchschnitte der Bronchien in Beginne der Affektion durch scharf gezeichnete schwefelgelbe Ringe angedeutet, deren âußerer Rand sehr regelmälig verläuft und sich sehr scharf von dem umgebenden, nicht erkrankten Lungenparenchym abgrenzt. Es ist auch sehr wichtig, zu berïcksichtigen, dall alle peribronchitischen Herde, einerlei, in welchem Entwickelungsstadium sie sich befinden, dieselbe gelbe Farbe zeigen. Der Prozeß hat nur wenig Neigung, sich auf das Lungengewebe fortzusetzen, während die Herde sich im Gegenteil nach der Mitte des Bronchiallumens hin vergrößern, das dann bald von den gelben Massen ausgefüllt wird. Selbst mikroskopisch findet man dann noch die umgebenden Alveolen frei und nur hier und da sieht man einen unregelmäßig knötchenförmigen Auswuchs des Ringes gegen dieselben andringen. Bei der Peribronchitis tuberculosa liegen die Verbältnisse doch ganz anders, die Gestalt der Herde ist unregelmäßig, sie ziehen leicht das umliegende Lungenparenchym in Mitleidenschaft, sie erheben sich mehr über das Niveau der Schnittfäche, sie haben nicht so sehr die Neigung $z u$ einem völligen Verschlusse der Bronchiallumina zu führen und itre Farbe ist je nach dem Entwickelungsstadium, in welchem sie sich befinden, aufer- 
ordentlich verschieden. Selbst wenn schon ausgesprochene Verkäsung vorhanden ist, sind die gelblichen Farbentöne doch ganz andere als bei der Aktinomykose und wenn man dann auch die übrigen geschilderten Verhältnisse berücksichtigt, dann wird die Differentialdiagnose zwischen Tuberkulose und Aktinomykose keine Schwierigkeiten machen.

Durch die Knötchen im Herzen scheinen keine Funktionsstörungen des Organs bedingt worden zu sein.

Den Verlauf der Krankheit stelle ich mir folgendermaßen vor: Die Einbruchspforte der Pilze hat wahrscheinlich im rechten Unterlappen gelegen, wo die beschriebene narbige Partie den ältesten Krankheitsherd repräsentiert und von hier sind sie, nachdem sich pleuritische Verwachsungen gebildet hatten, in diesen und zwischen Zwerchfell und Brustwand nach unten gedrungen, um dort, an der Insertion des Zwerchfells, das letztere zu durchbrechen und in die Leber ein- und nach aufwärts zu dringen; ferner hat oben ein Einbruch durch das Zwerchfell in die Leber stattgefunden. Wenn der narbige Herd im rechten Unterlappen tatsächlich, wie ich glauben möchte, der erste Krankheitsherd gewesen ist, so liegt es nahe, an das Verhalten von Fremdkörpern in der Lunge zu denken, welche doch auch gewöhnlich in den rechten Unterlappen gelangen, und anzunehmen, daß die infizierende Masse mindestens ein sichtbares Körnchen gewesen sei, bei dem die gleichen Verhältnisse wie bei Fremdkörpern die erste Lokalisation veranlaßt haben. Später sind dann freigewordene Keime in die oberen Bronchien gelangt und haben peribronchitische Veränderungen hervorgerufen.

Eine sehr vollständige Liste von Arbeiten über Aktinomykose findet sich in „Ergebnisse der allgemeinen Pathologie und pathologischen Anatomie des Menschen und der Tiere", von Lubarsch und Ostertag, 1898, doch möchte ich derselben noch die in Nordamerika publizierten Fälle, von denen ich nur denjenigen von Bodamer erwähnt finde, hinzufügen.

\section{Literatur.}

1. A. Schirmer: Chicago Med. Journ. and Examiner. Vol. VIII, S. 354. 1886. (Lokalisation: Am Kiefer.) Wahrscheinlich auch Aktinomykose der Lungen, denn Pilzdrüsen wurden im Sputum gefunden. 
2. A. J. Ochsner: Journal of the American Medical Association 1886. S. 608. (Lokalisation im linken Antrum Highmori.) No. 2. (Lokalisation: Rechte Tewporalgegend.)

3. Geo. A. Bodamer: "The Pathology of Actinomycosis, With Record of cases and Experiments". The Journal of comparative Medicine and Surgery. April 1889. Vol. 10.

4. J. M. Byron: "A case of Actinomycosis in Man“. New York Med. Journal. Dec. 28th 1889, S. 716. (Peripleuritis actinomycotica". Lokalisation: In der rechten mitttleren Axillarlinie, zwischen der 5. und 7. Rippe.

5. J. B. Murphy: The Chicago Medical Recorder. Febr, 1892. Actinomycosis Hominis, With Report of five cases

Fall 1. Lokalisation: Linker Unterkiefer.

$\begin{array}{llll} & 2 . & & \text { Rechter } \\ & 3 . & n & \\ & 4 . & & \text { Peritonaeum. } \\ " & 5 . & \# & \text { Unterkiefer. }\end{array}$

6. Lyman Brown: "A case of Actinomycosis“, Chicago Medical Recorder. October 1894. No. 4, S. 251. (Lokalisation: Linker Unterkiefer.)

7. Mixter: Boston Med. and Surg. Journ. Vol. OXXXII. No. 13. March 28th 1895. Lokalisation: Unter dem Nabel.

8. Parker Syms: „Actinomycosis", Annals of Surgery, F. 1897. Lokalisation: Abdomen.

\section{XVII. \\ Ein Fall von Sirenenbildung. (Sympus apus).}

(Aus der Prosektur des Nikolai-Stadtkrankenbauses zu Rostow a. D.)

Von

Prosektor S. Abramow und Dr. M. Rjesanow.

(Mit 6 Textfiguren.)

Im Sommer 1901 gebar eine vollkommen gesunde Frau in der geburtshilflichen Abteilung des Rostowsehen a. D. Stadtkrankenhauses eine fußlose Sirenenbildung (Sympus apus). 